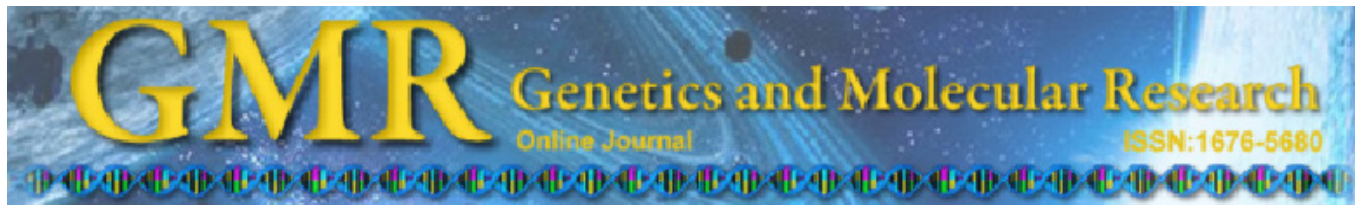

\title{
A novel and efficient strategy for practical identification of tomato (Solanum lycopersicon) varieties using modified RAPD fingerprints
}

\author{
N.K. Korir ${ }^{1,2}$, X.Y. Li ${ }^{1,3}$, X.P. Leng ${ }^{1}$, Z. Wu ${ }^{1}$, C. Wang ${ }^{1}$ and J.G. Fang ${ }^{1}$ \\ ${ }^{1}$ College of Horticulture, Nanjing Agricultural University, Weigang, Nanjing, \\ China \\ ${ }^{2}$ Department of Agricultural Technology, Kenyatta University, Nairobi, Kenya \\ ${ }^{3}$ Zhejiang Academy of Agricultural Sciences, Hangzhou, China
}

Corresponding author: J.G. Fang

E-mail: fanggg@njau.edu.cn

Genet. Mol. Res. 12 (2): 1816-1828 (2013)

Received October 17, 2012

Accepted March 2, 2013

Published June 11, 2013

DOI http://dx.doi.org/10.4238/2013.June.11.2

\begin{abstract}
Tomato breeding and variety development have led to the generation of a large number of varieties in many countries worldwide. This has created a growing and urgent need for an improved strategy for genotyping and identification since the traditional methods based on phenotype are growing unreliable. DNA markers could provide distinct benefits in tomato variety identification; however, DNA fingerprint analyses have not made DNA marker data readily usable for identification of varieties in tomato and other crops. A manual cultivar and/or variety identification diagram (MCID) strategy has been developed and has been found to make DNA markers more usable for the identification of genotyped plant individuals. We adopted this strategy, using modified RAPD markers to identify 42 tomato varieties from different geographical origins and seed merchants. All of the varieties were clearly separated and individually identified by reproducible fingerprints of only 6 RAPD primers. The tomato MCID that is generated is usable for the identification of any two or more tomato varieties. In addition, fewer primers can be used to make a distinction between varieties using this approach, since the selected fingerprints from each primer are used after they have been generated. The information in this
\end{abstract}


first version of the tomato MCID can be enriched through identification and incorporation of more varieties and adaptation to other molecular markers in order to provide a more comprehensive tomato variety identification service for the horticultural industry.

Key words: Tomato varieties; Manual cultivar identification diagram; RAPD

\section{INTRODUCTION}

Tomato is one of the most important fruit/vegetable crops in the world. Its production in 2010 was more than 145.5 million tons, harvested from over 4.3 million hectares at an average yield of $336 \mathrm{hg} / \mathrm{ha}$ (FAO, 2012). There are more than 7500 tomato varieties successfully bred and grown for various purposes worldwide, and plant variety registration bodies in different countries keep records of most of these varieties. Although each tomato variety has unique characters of interest to breeders and other researchers as well as growers, cases of synonyms and homonyms abound in the tomato industry just like in other crops, and this is an important problem that should not be overlooked but rather should be addressed (Korir et al., 2012). With the evolution of the tomato industry, the development and adoption of new varieties have gained popularity, thus making the breeding of and research on tomato varieties more and more important. This in turn has created a need for their accurate and rapid identification by both breeders and key commercial and regulatory players in the tomato value chain. Even though there have been some reports on tomato variety identification using molecular markers (Patil et al., 2010; Abd El-Hady et al., 2010; Huh et al., 2011; Ezekiel et al., 2011), the phylogenetic tree-based dendrograms of the varieties generated from cluster analysis of the DNA banding patterns have not actually made the identification of tomato varieties easy and practical, other than indicating the genetic diversity levels of the plant individuals. This therefore calls for strategies and methods that can make the identification of tomato varieties reliable, easy, and referable since it is vital for the tomato seed and nursery industries, as well as important to growers and researchers for purposes of protecting plant patents and providing genetically uniform plants.

Molecular markers are valuable due to the fact that they are not influenced by the environment and can therefore provide a powerful tool for appropriate categorization of crop genotypes including varieties and cultivars. Many DNA-based markers, such as RFLP (Williams et al., 1991), RAPD (Williams et al., 1990), SSR (Powell et al., 1996), I-SSRs (Zietkiewicz et al., 1994), AFLP (Vos et al., 1995), SNPs, SAMPL (Morgante and Vogel, 1994), M-AFLP (Albertini et al., 2003), SRAP (Li and Quiros, 2001), CAPS (Williams et al., 1991), and SCoT (Collard and Mackill, 2009), have been developed and used in studies on genetic diversity, fingerprinting and origins of cultivars in many crops (Fang et al., 2005; D'Onofrio et al., 2009; Melgarejo et al., 2009; Papp et al., 2010). Among these markers, the dominant RAPD marker (Williams et al., 1990) is generally accepted for basic genotype characterization, genome analysis and gene mapping in various species due to its simplicity, speed and efficiency, ability to detect relatively small amounts of genetic variation and non-requirement of prior information on the genome. Since the approach requires no prior knowledge of the genome that is being analyzed, it can be readily employed across 
species using universal primers. The major drawback of RAPD markers is that the profiling is dependent on the reaction conditions which may vary between different laboratories, thus making it less sensitive and reproducible (Rajput et al., 2005; Agarwal et al., 2008). This problem can, however, be solved by optimization of the RAPD technique by choosing 11 nucleotide primers and strict screening of PCR annealing temperature for each primer before RAPD is employed in fingerprinting plants (Welsh and McClelland, 1990; Penner et al., 1993). In addition, the results from RAPD can be analyzed and presented in different ways ranging from clustering, dendograms, phylogenetic trees, and cultivar identification diagrams, among others. Depending on the strategy adopted, all these can in turn make RAPD a useful technique in plant cultivar and variety identification to varying degrees as shown by recent studies using the manual cultivar identification diagram (MCID) strategy in different crops (Zhang et al., 2011; Zhao et al., 2011; Wang et al., 2011; Li et al., 2011). RAPD markers have also been successfully applied in determining genetic relationships in vegetables such as Capsicum (Bhadragoudar and Patil, 2011), broccoli (Lu et al., 2009), radish (Liu et al., 2008), and curcurbits (Dey et al., 2006; Sureja et al., 2006; Verma et al., 2007), among other crops.

The application of RAPD markers in the practical identification of tomato varieties would be of great scientific and commercial value in the tomato industry, and if successful and broadly accepted, it can be further replicated in other important vegetable, ornamental, fruit, and cereal crops. The MCID strategy developed by Zhang et al. (2011) has been found to make DNA markers more usable in the identification of genotyped plant individuals of different species (Zhao et al., 2011; Wang et al., 2011; Li et al., 2011), and therefore, the main objective of this study was to explore the feasibility of using the MCID strategy and employing modified RAPD to identify tomato varieties in a practicable, efficient, recordable, and referable manner. A total of 42 selected tomato varieties were identified and a referable cultivar identification diagram (CID) of these tomato varieties developed. The CID may be employed as a reference table with greater advantages regarding ease of use, workability, and flexibility, whereby new varieties can be readily added as their data become available. Furthermore, this CID will add precious information and buttress the theoretical basis for the identification of varieties and cultivars, genetic diversity analysis and genetic improvement of crops at the molecular level, as well as being a potentially essential tool in testing new varieties for DUS (distinctness, uniformity and stability) before granting protection (Lu et al., 2009).

\section{MATERIAL AND METHODS}

\section{Plant materials and genomic DNA extraction}

Seeds from a total of 42 tomato genotypes (Table 1) with geographically distributed origins were collected from research centers, seed companies and seed shops in China and Kenya and planted in a growth room for 3 weeks before extraction of total genomic DNA for use in this study. The choice of varieties was based on popularity in various markets and apparent claims of similarity in some varieties by growers and other players in the horticultural industry. Total genomic DNA of each variety was extracted from young leaves using the modified cetyltrimethylammonium bromide (CTAB) method (Murray and Thompson, 1980; Bousquet et al., 1990), and the extracted DNA was diluted to a final concentration of $30 \mathrm{ng} / \mu \mathrm{L}$ with $1 \mathrm{X}$ TE buffer and stored at $-20^{\circ} \mathrm{C}$ until further use. 
Table 1. Cultivars identified using the tomato manual cultivar identification diagram and their origins.

\begin{tabular}{|c|c|c|}
\hline Code & Cultivar name & Origin \\
\hline 1 & Jingdanfenyu 2 & Beijing, China \\
\hline 2 & Caiyu 3 & Beijing, China \\
\hline 3 & Ying fen 8 & Beijing, China \\
\hline 4 & Xian ke 1 & Beijing, China \\
\hline 5 & Jiang shu 14 & Jiangsu, China \\
\hline 6 & Xian ke 6 & Beijing, China \\
\hline 7 & Cai yu 1 & Beijing, China \\
\hline 8 & Cai yu 2 & Beijing, China \\
\hline 9 & Sheng xing guo & Jiangsu, China \\
\hline 10 & Qiu zhan 16 & Beijing, China \\
\hline 11 & Jia hong 4 & Beijing, China \\
\hline 12 & Jiahong 5 & Beijing, China \\
\hline 13 & Suhong 2003 & Jiangsu, China \\
\hline 14 & Xinyanlvcaiqiu & Heilongjiang, China \\
\hline 15 & Jingdanhuangyu & Beijing, China \\
\hline 16 & Eden & Kenya \\
\hline 17 & CAL-J & Kenya \\
\hline 18 & Jingdanfenyu 1 & Beijing, China \\
\hline 19 & Jingdan 1 & Beijing, China \\
\hline 20 & Huangying 1 & Beijing, China \\
\hline 21 & Jinman & Beijing, China \\
\hline 22 & Tylka & Kenya \\
\hline 23 & Assila & Kenya \\
\hline 24 & Cherry sun gold & USA \\
\hline 25 & Zhaoyan 296 & Jiangsu, China \\
\hline 26 & Bonnie Besst & USA \\
\hline 27 & Luomanna & Holland \\
\hline 28 & German Johnson & USA \\
\hline 29 & Jina & Holland \\
\hline 30 & Jiali & Holland \\
\hline 31 & Qinghuangfentianshi & Uknown \\
\hline 32 & Hena & Holland \\
\hline 33 & Cherry super sweet 100 & USA \\
\hline 34 & Hezuo 908 & Shanghai, China \\
\hline 35 & Gailiangkaluoyi & Holland \\
\hline 36 & Zhaoyan 269 & Jiangsu \\
\hline 37 & Dihuanghuangying tao & Uknown \\
\hline 38 & Beef steak & USA \\
\hline 39 & Fushi 3 & Holland \\
\hline 40 & Cherry Gardeners Delight & USA \\
\hline 41 & Yellow Pear & USA \\
\hline 42 & Fenguan & Jiangsu \\
\hline
\end{tabular}

\section{Amplification of RAPD markers}

The reaction mixture (final volume of $25 \mu \mathrm{L}$ ) contained $2.5 \mu \mathrm{L} 10 \mathrm{X}$ buffer, $2.0 \mu \mathrm{L} 2.5$ $\mathrm{mM}$ dNTP, $1.5 \mu \mathrm{L} 25 \mathrm{mM} \mathrm{MgCl}_{2}, 1.0 \mu \mathrm{L} 1.0 \mu \mathrm{M}$ primer, $0.10 \mu \mathrm{L} 5 \mathrm{U} / \mu \mathrm{L}$ rTaq Polymerase Dynazyme, and $60 \mathrm{ng}$ genomic DNA. Amplification reactions were performed according to Williams et al. (1990), with minor modifications. PCR was carried out in an Autorisierter Thermocycler (Eppendorf, Hamburg, Germany), using the following procedure: pre-denaturation at $94^{\circ} \mathrm{C}$ for $5 \mathrm{~min}$, and then 42 cycles of denaturation at $94^{\circ} \mathrm{C}$ for $2 \mathrm{~min}$, annealing at $35^{\circ}-45^{\circ} \mathrm{C}$ for $1 \mathrm{~min}$, and extension at $72^{\circ} \mathrm{C}$ for $1 \mathrm{~min}$.

\section{Analysis with modified RAPD}

A set of 60 11-mer RAPD primers were optimized and screened in this study, and re- 
producible polymorphic bands were obtained with 12 randomly selected primers. Reliability of the fragments on the gels was enhanced by scoring only those that were reproducible. At the end, only 6 primers (Table 2) that showed well-resolved and reproducible bands were further analyzed, and the rest declared redundant. All PCR products were detected on $1.6 \%(\mathrm{w} / \mathrm{v})$ agarose gels in $1 \mathrm{X}$ TAE (0.04 M Tris-acetate, $0.001 \mathrm{M}$ EDTA, pH 8.0) buffer at $100 \mathrm{~V}$. The gels were stained with $0.5 \mu \mathrm{g} / \mathrm{mL}$ ethidium bromide and photographed under ultraviolet light. Polymorphic bands among the varieties were observed from the photographs. All amplifications were performed thrice to obtain reproducible and clear banding patterns.

Table 2. Primers selected for identification of 42 tomato varieties.

\begin{tabular}{ll}
\hline Primer code & Sequence $\left(5^{\prime} \rightarrow 3^{\prime}\right)$ \\
\hline Y-21 & GGACCCAACCA \\
Y-22 & GGACCCAACCT \\
Y-27 & GTGTGCCCAA \\
Y-35 & AAGCCTCGTCG \\
Y-53 & TGGTGGCGTTG \\
Y-59 & ACCCCCGACTG \\
\hline
\end{tabular}

\section{Data analysis}

Only clear and directly recognizable bands on the gel photographs were manually chosen and scored for each variety and each primer. Varieties with a distinct band in the fingerprint generated from one primer were separated apart, while varieties sharing the same banding pattern were separated into the same subgroup, and the rest into another subgroup. Following these criteria, all 42 tomato varieties were gradually and completely separated from each other as additional primers were used. A CID was then prepared based on the stages of separation of the varieties and on the primers and banding patterns used at each stage.

\section{Assessment of use and workability of the CID in tomato varieties}

There was a critical need to verify the workability of the CID and demonstrate how it can be employed in the future. To achieve this, several tomato varieties were indiscriminately chosen from inside and outside the defined groups on the CID and subjected to new identification following the same procedure as used above. The corresponding primers to be used for the separation of each group were simply picked out from the diagram.

\section{RESULTS}

\section{Tomato variety identification}

To establish the suitability of the RAPD marker in identifying the tomato varieties, a set of 60 RAPD primers were screened and the optimal annealing temperature for each primer determined based on the value and reproducibility of banding patterns. After all primers were tested, each of the 42 tomato varieties could be fully separated and individually identified by the systematic use of only six primers (Table 2). An outstanding illustration of this identifica- 
tion, based on the RAPD pattern, is that obtained with primer Y35 (Figure 1A). Primer Y35 was the first to be screened out and used to separate the 42 tomato varieties. The electrophoresis results showed that two varieties denoted as 9 and 41 (lane numbers corresponding to the codes in Table 1) both generated a uniform, clear and reproducible 200-bp band, which was absent in all the other varieties (Figure 1A). When this special band is selected for variety identification, these two varieties can be instantly recognized. Similarly, three tomato varieties denoted as 18,20 and 21 could be selected out of the other varieties based on the specific presence of a 1300-bp band and absence of 750- and 200-bp bands, a situation that is unique to these three varieties (Figure 1A). The remaining varieties were then separated into two groups according to two specific bands of 750 and $1300 \mathrm{bp}$. Thirty-three varieties $(1,3,4,5,6,7,8,10,11,12,13$, $14,15,16,17,19,22,23,24,25,26,27,28,29,30,33,34,35,36,37,38,39$, and 42) generated both bands, while 4 varieties $(2,31,32$, and 40$)$ generated only one specific band of 1300 bp (Figure 1A). A second primer Y53 was chosen to differentiate varieties in the new groups based on the presence or absence of a 700-bp band, leading to the creation of 4 new clusters of varieties and complete separation of varieties 20 and 40. In view of the fact that the members in the remaining four clusters related to primer Y53 could not be conclusively differentiated, generation or not of a 1200-bp band by primer Y27 was used to further distinguish varieties within these groups, thus creating 5 new clusters and complete separation of varieties 18, 21 and 32 at this step (Figure 1B and C).
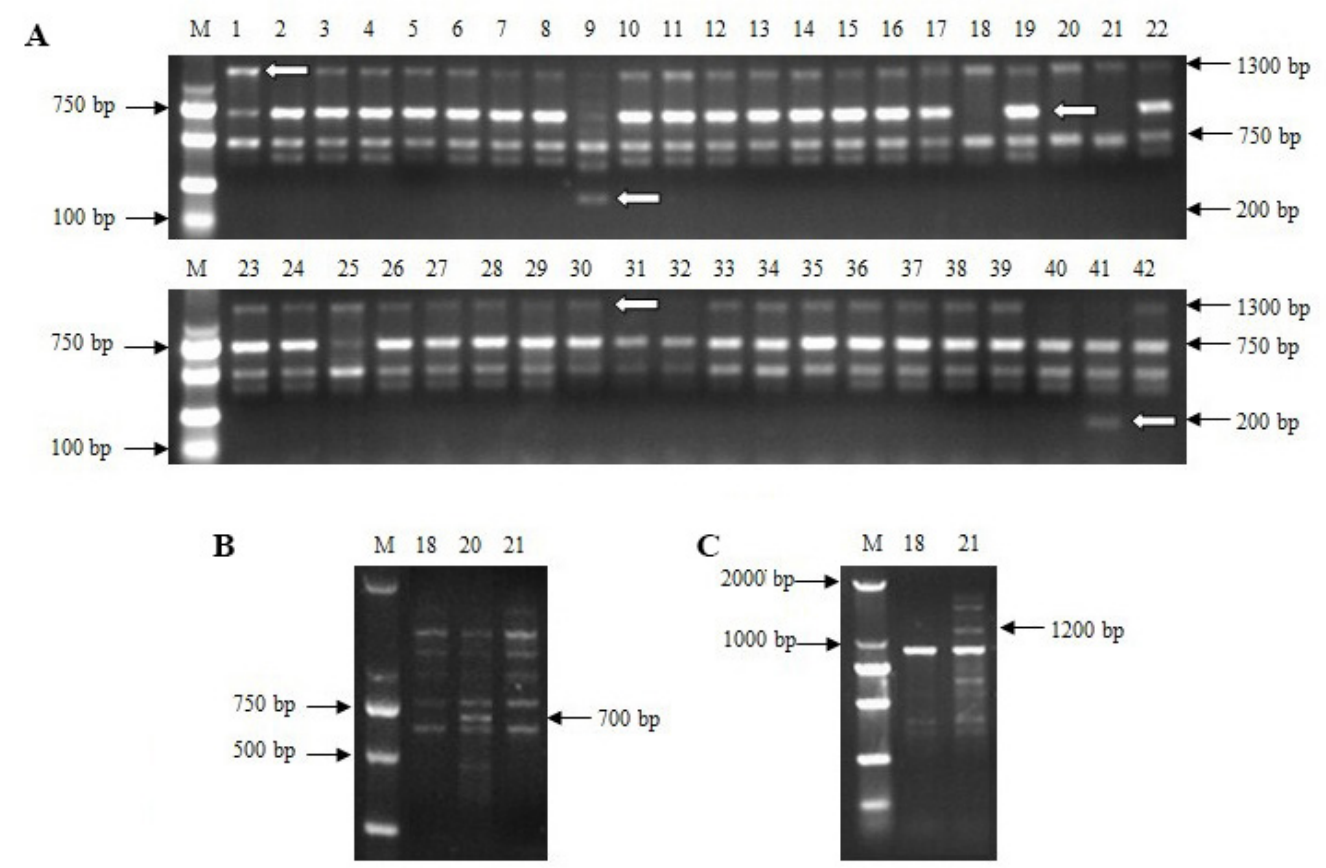

Figure 1. Banding patterns of 42 tomato varieties obtained with modified RAPD primers. A. B. C. RAPD profiles obtained with primers Y35, Y53 and Y27, respectively. Horizontal arrows indicate the specific bands used. The lane numbers correspond to the codes in Table 1 . Lane $M=$ DNA size marker. 
Varieties in the 5 new groups were subjected to similar separation using primer Y59 followed by primers Y21 and Y22 (Figure 2). Primer Y59 provides a good example of a primer that generates several polymorphic bands, meaning that many varieties could be successfully differentiated from each other at this stage (Figure 2). By adopting this strategy, the initial group of 42 tomato varieties could be totally differentiated from each other using only 6 of the original 60 primers, and a tomato CID constructed (Figure 2). The sizes and presence or absence of the polymorphic bands used for separating the varieties are indicated at each corresponding elimination step and the varieties completely separated at each disconnection shown by names in bolded text. All primers and polymorphic bands shown on the CID can provide the information needed for the identification of any of the varieties as planned, thus making this CID practically workable and referable in tomato breeding, propagation, production, and even processing industries.

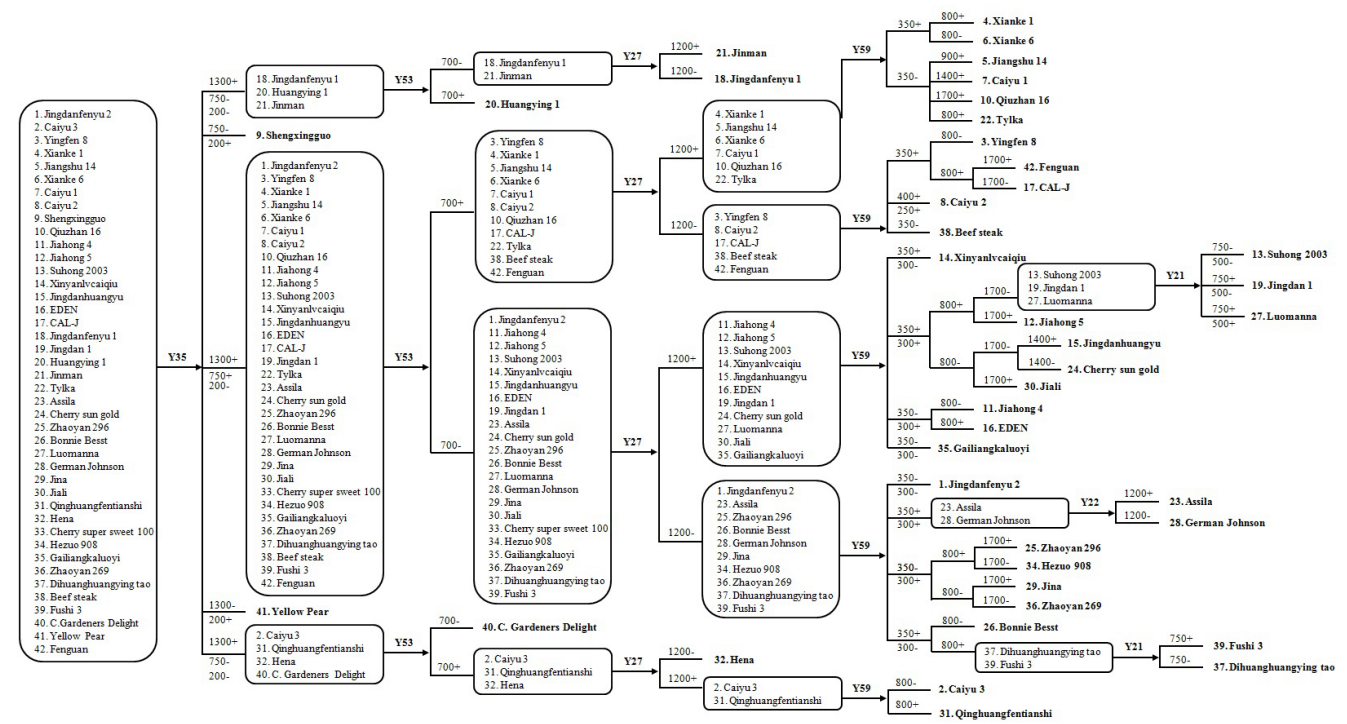

Figure 2. A manual cultivar identification diagram (MCID) of 42 tomato varieties based on 7 RAPD primers. The number above each horizontal line in the figure means the size of the band in bp; $(+)=$ band present; $(-)=$ band absent. The names of varieties in bold mean that these varieties were separated out at that point in the MCID. The varieties denoted as $1,3,4,5,6,10,11,17,19,25,28$, and 36 were randomly selected and used in validation of the MCID.

\section{Assessment of utilization and workability of the MCID for variety identification}

The purpose of this research was to determine the possibility of using modified RAPD markers to identify tomato varieties in a simple and replicable strategy. In addition, we sought to generate a referable tomato CID for the identification of varieties and their products in the tomato industry and for possible use in ensuring the protection of breeder/ owner rights. Reproducibility and referability are critical pillars that determine the success of a scientific strategy, and therefore the CID was subjected to tests to verify the utilization, workability and efficiency of the diagram in variety identification. Twelve tomato varieties denoted as $17,36,4,19,11,28,3,10,5,6,1$, and 25 were picked at random from inside and 
outside the different groups or subgroups in the CID and used to authenticate and illustrate the scientific aspects of this method. From the location of these varieties in the CID, it was quite easy to identify the primers needed for separating them. For these 12 varieties, the primers Y53, Y27 and Y59 (Figure 2) were chosen and used, where the final PCR results clearly showed that these tomato varieties could be identified by the specific bands as anticipated in Figure 2. For example, primer Y53 was used to amplify the genomic sequences of '17' and '36' and '4' and '19' (Figure 3A), where a specific band of 700 bp was used for identification. Primer Y27 was used to amplify the genomic sequence of ' 11 ' and ' 28 ', or ' 3 ' and '10' (Figure 3B), where the specific band whose fragment size was about $1200 \mathrm{bp}$ was used for identification. Similarly, to separate ' 5 ' and ' 6 ', or ' 1 ' and ' 25 ', primer Y59 and a $350-$ or $300-b p$ band were needed. This confirmation test proved that this method is both precise and reliable.

$\mathbf{A}$

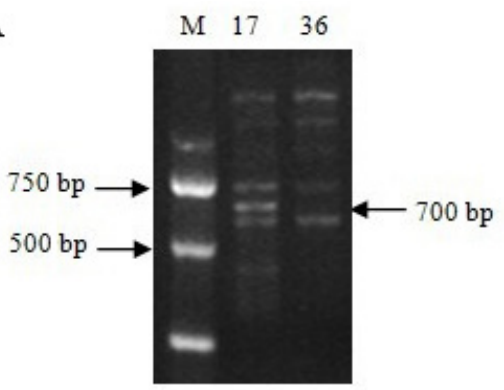

B

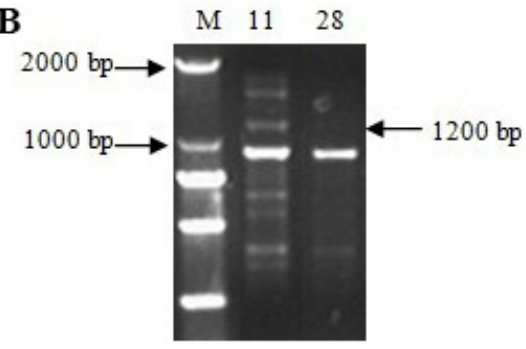

C

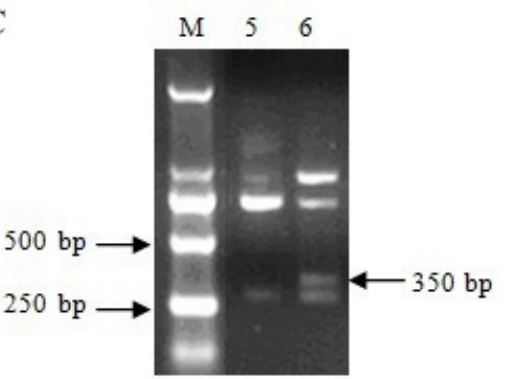

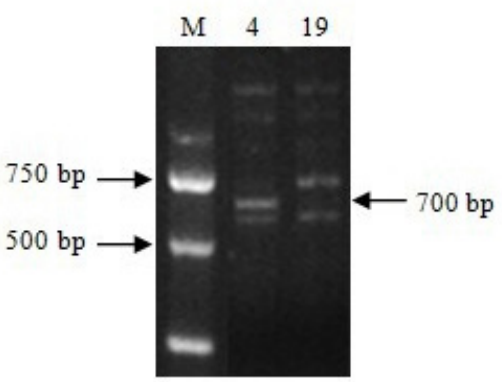
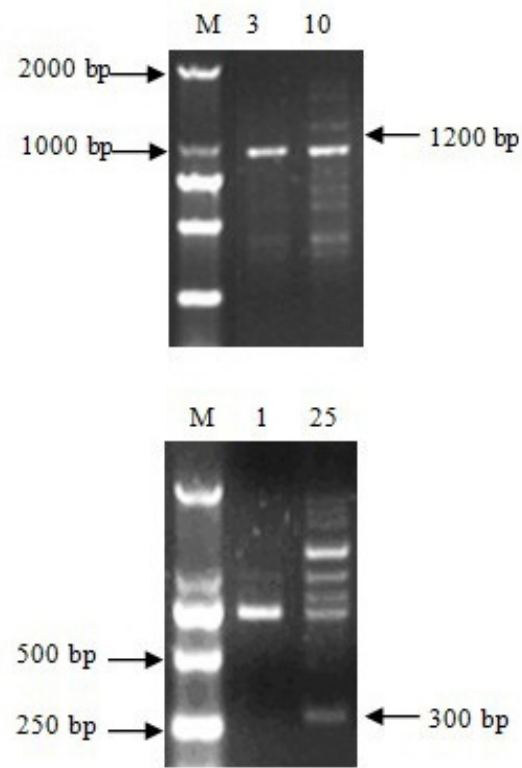

Figure 3. RAPD profiles obtained with optimized RAPD primers during verification of the manual cultivar identification diagram. Horizontal arrows indicate the specific bands. The lane numbers correspond to the code in Table 1. Lane $M=$ DNA size marker. A. Obtained with Y53 primer. B. Obtained with primer Y27. C. Obtained with primer Y59. 


\section{DISCUSSION}

The use of molecular markers serves as a modern and suitable approach to cultivar and variety identification, since it is more rapid and cost-effective (Korir et al., 2012). Different molecular marker techniques have been used in plant population genetics and identification, phylogenetic and biodiversity studies, analysis of recombination frequencies between genotypes, identification of genes for important agricultural traits and marker-assisted selection (Vishwanath et al., 2010; Sonah et al., 2011). Molecular biology methods, especially DNA fingerprinting techniques, have promising applications in the identification of plant genotypes including varieties and cultivars. Indeed, such tools would be very useful for breeders, growers and the general industry, since fraud is common in variety/cultivar development and usage. These forms of fraud consist of selling cultivars that are propagated without permission or marketing plants with a wrong cultivar name (usually a stolen name), with both types of fraud sometimes being observed in the same case. Such deceptions obviously cause important economic losses to plant breeders, who invest a considerable amount of money in the creation of new varieties and/or cultivars (Agarwal et al., 2008).

DNA-based molecular markers have served as versatile tools in the fields of taxonomy, genetic engineering, marker-assisted selection, variety and cultivar identification as well as variability studies. These markers are found in abundance and are more precise, thus providing an opportunity for direct comparison of genetic materials. In addition, molecular markers are not affected by different environmental conditions or the stages of growth and development of plants (Reddy et al., 2002). In spite of these benefits and potential, DNA markers have not been easily and widely used in genotyping plant species, varieties or cultivars. The gravity of this situation is indicated by the lingering doubt among plant scientists and seed trade associations on whether DNA markers can be readily used in the identification of plant varieties and cultivars and in evaluation of DUS. It has been shown that if uniformity (or stability) tests were carried out using the same set of molecular markers as used for the distinction test, uniform varieties for morphological and agronomic traits could be rejected because of lack of molecular uniformity. Breeding companies do not want this because it would be more expensive to develop new molecularly uniform varieties. However, plant variety protection agencies and breeding companies would like to count on molecular markers for distinction tests given that it is easier and quicker to check differences at the molecular level than at the morphological level. Therefore, as shown by Bernet et al. (2003) on Cucumis sativus, there are conflicts of interests, whereby molecular markers can be very useful for distinction tests but their utilization in uniformity and stability tests would be very expensive for plant variety protection agencies and breeding companies in some crop species.

Few approaches including phylogenetic clustering and DNA fingerprinting have been developed to utilize DNA markers in plant cultivar and variety identification. However, none of these have the features that would make plant variety identification easy, practical and referable. The clusters formed in phylogenetic trees do not tell which information is referable in the identification of plant samples, while fingerprinting does not show all the fingerprints from many varieties together for identification. These weaknesses may be attributed to the fact that no analysis connects the information of DNA fingerprints with varieties in an uncomplicated and comprehensible manner. The relatively new approach employed in this study can use DNA markers efficiently to distinguish the varieties as desired. It has been successfully used 
to identify cultivars in grapes, citrus, peaches, plums, and pears (Zhang et al., 2011; Zhao et al., 2011; Wang et al., 2011; Li et al., 2011), and there are several ongoing studies aimed at using this strategy on other crops and employing a wider array of molecular markers. This study is the first attempt at utilizing the strategy to identify varieties in tomato, a vegetable crop. It has the advantages of less cost and time and objectivity, among others. The MCID strategy brings out the power of DNA markers in plant variety and cultivar identification activities, and it shows that polymorphic bands of each primer can be used to gradually distinguish every species and individual plants, from which a CID can be finally constructed for further use in identifying these plant genotypes.

The classification of tomato genotypes is sometimes wrought with debate, partly because of cases of inter-varietal hybridization, which creates several intermediate types, and sometimes blurs the distinction between these varieties. The presence of homonyms or synonyms within existing tomato varieties being sold in the markets shows the need to properly identify wild and cultivated tomato varieties and cultivars for conservation and use of these germplasm resources as well as in plant variety protection. The ability to distinguish varieties could be greatly enhanced by using appropriate molecular markers (Patil et al., 2010; Ezekiel et al., 2011; Korir et al., 2012). The well-documented advantages of optimized RAPD markers make it the technique of choice for the initial phase of this study. The key objective was to determine the possibility of using RAPD markers to distinguish 42 tomato varieties, which is one way of utilizing DNA fingerprints in identifying plant varieties and cultivars. In addition we sought to test the viability and reproducibility of an MCID strategy for the separation of tomato varieties. This methodology could also be considered as a universal strategy to use in distinguishing varieties/cultivars and seed samples in other plant species as reported by Li et al. (2011).

Using the tomato MCID approach, only 6 RAPD primers were used to differentiate all 42 tomato varieties sampled in this study. This system is both handy and speedy for the user. Although a single primer cannot distinguish all varieties tested simultaneously, this strategy has advantages over earlier methods of tomato variety identification. In addition, this study provides new evidence of speedy identification of tomato varieties and additional uses of RAPD markers. The revealing MCID (Figure 2) of the tomato varieties can readily show the primers needed to separate any of the 42 varieties. In essence, any two or more tomato varieties can be distinguished by the use of one specific primer. This can be exemplified by the varieties ' 18 ' and ' 21 ', which can be distinguished by the use of primer Y27 (Figure 3). When PCR amplification shows a special band of $1200 \mathrm{bp}$, the variety is ' 21 '; otherwise it is ' 18 '. Any other two varieties can also be distinguished following this same principle. If and when new tomato varieties need to be identified or are bred and released, the set of 6 primers chosen in this study can be used for PCR analysis of DNA from these new varieties, and the PCR banding patterns can readily indicate where to position the new varieties in the CID. If they cannot be separated from the 42 already identified using the 6 primers, new primers can be designed, screened and used for separation, and these new varieties can then be positioned on the CID. This kind of variety identification procedure is not mind-numbing and can generate a bigger CID of tomato varieties in the long run, which makes it a useful resource, needed in the wider tomato industry. It should be pointed out that although such a method may not precisely reflect genetic relationships among the varieties, the genetic distance between varieties separated using the first primer is theoretically always larger than the distance between varieties 
separated by the last primer (Wang et al., 2011). This strategy is indeed a great addition to the important exercise of plant variety identification and will be quite useful in the protection of cultivar-rights and early identification.

This is the first study reporting optimization of the RAPD technique by choosing 11 nucleotide primers and strict screening of PCR annealing temperature for each primer before using RAPD in sequential fingerprinting of tomato varieties. Replication and experimental verification done to confirm the reliability of this idea and strategy gave reasonable results for the selected primers. Therefore, this study further confirms the potential of utilizing DNA markers even in plant species, without requiring a genetic linkage map and/or any DNA sequence information to distinguish the varieties, cultivars or any other genotypic grouping. The MCID plus RAPD marker technique was found to be effective and convenient in selecting markers in fruit trees and it has great potential in other crop plants. Furthermore, the polymorphic bands generated may be developed into special molecular markers for the identification of varieties/cultivars in future. The generation of a readable and referable CID is significant since such a CID can be constructed and used in the identification of related plant species. This study is a continuation of research on this method, and as it progresses, this stratagem in concert with other molecular markers can be used to develop a reference table for each species among organisms of interest, which in turn can provide the information needed to separate varieties, cultivars, etc., as desired. Different markers and their applications should be tested to ascertain their suitability for use in variety identification, since some markers have been found to be occasionally unstable, calling for measures to mitigate this. A good example is the use of stable 11-mer RAPD primers in our study instead of the unstable 10-mer options. The banding patterns from the 11-mer primers were reproducible from the replicates and from the exercise to verify workability of the CID strategy.

We, therefore, conclude that this approach of using RAPD markers and an MCID is rapid and simple and produces reliable results, since it was possible to demonstrate that a small grouping of primers can be used to distinguish between large numbers of tomato varieties. However, it is recommended that this strategy be also used with more varieties and other markers so as to generate a wider database and give a comparative recommendation.

\section{ACKNOWLEDGMENTS}

Research supported by Fundamental Research Funds for the Central Universities.

\section{Conflicts of interest}

The authors have no declarations to make.

\section{REFERENCES}

Abd El-Hady EAA, Haiba AAA, Abd El-Hamid NR and Rizkalla AA (2010). Phylogenetic diversity and relationships of some tomato varieties by electrophoretic protein and RAPD analysis. J. Am. Sci. 6: 434-441.

Agarwal M, Shrivastava N and Padh H (2008). Advances in molecular marker techniques and their applications in plant sciences. Plant Cell Rep. 27: 617-631.

Albertini E, Porceddu A, Marconi G, Barcaccia G, et al. (2003). Microsatellite-AFLP for genetic mapping of complex polyploids. Genome 46: 824-832. 
Bernet GP, Bramardi S, Calvache D, Carbonell EA, et al. (2003). Applicability of molecular markers in the context of protection of new varieties of cucumber. Plant Breed. 122: 146-152.

Bhadragoudar MR and Patil CG (2011). Assessment of genetic diversity among Capsicum annuит L. genotypes using RAPD markers. Afr. J. Biotechnol. 10: 17477-17483.

Bousquet J, Simon L and Lalonde M (1990). DNA amplification from vegetative and sexual tissues of tree using polymerase chain reaction. Can. J. For. Res. 20: 254-257.

Collard BCY and Mackill DJ (2009). Start codon targeted (SCoT) polymorphism: A simple, novel DNA marker technique for generating gene-targeted markers in plants. Plant Mol. Biol. Rep. 27: 86-93.

D’Onofrio C, Lorenzis G, Giordani T, Natali L, et al. (2009). Retrotransposon-based molecular markers in grapevine species and cultivars identification and phylogenetic analysis. Acta Hort. 827: 45-52.

Dey SS, Singh AK, Chandel D and Behera TK (2006). Genetic diversity of bitter gourd (Momordica charantia L) genotypes revealed by RAPD markers and agronomic traits. Sci. Hortic. 109: 21-28.

Ezekiel CN, Nwangburuka CC, Ajibade OA and Odebode AC (2011). Genetic diversity in 14 tomato (Lycopersicon esculentum Mill.) varieties in Nigerian markets by RAPD-PCR technique. Afr. J. Biotechnol. 10: 4961-4967.

Fang J, Qiao Y, Zhang Z and Chao CT (2005). Genotyping fruiting-mei (Prunus mume Sieb. et Zucc.) cultivars using amplified fragment length polymorphism markers. HortScience 42: 325-328.

FAO (2012). Agricultural Statistics of the Food and Agriculture Organization of the United Nations. FAOSTAT, FAO Statistics Division, Rome. Available at [www.fao.org]. Accessed April 9, 2012.

Huh MK, Youn SJ and Kang SC (2011). Identification and genetic diversity of Korean tomato cultivars by RAPD markers. J. Life Sci. 21: 15-21.

Korir NK, Han J, Shangguan L, Wang C, et al. (2012). Plant variety and cultivar identification: advances and prospects. Crit. Rev. Biotechnol. [Epub ahead of print].

Li G and Quiros CF (2001). Sequence-related amplified polymorphism (SRAP), a new marker system based on a simple PCR reaction: its application to mapping and gene tagging in Brassica. Theor. Appl. Genet. 103: 455-461.

Li XY, Wang C, Yang G, Li XD, et al. (2011). Employment of a new strategy for identification of Prunus mume cultivars using random amplified polymorphic deoxyribonucleic acid (RAPD) markers. Afr. J. Plant Sci. 5: 500-509.

Liu LW, Zhao LP, Gong YQ, Wang MX, et al. (2008). DNA fingerprinting and genetic diversity analysis of late-bolting radish cultivars with RAPD, ISSR and SRAP markers. Sci. Hort. 116: 240-247.

Lu X, Liu L, Gong Y, Zhao L, et al. (2009). Cultivar identification and genetic diversity analysis of broccoli and its related species with RAPD and ISSR markers. Sci. Hort. 122: 645-648.

Melgarejo P, Martcnez JJ, HerncLndez Fca, Martcnez R, et al. (2009). Cultivar identification using 18S-28S rDNA intergenic spacer-RFLP in pomegranate (Punica granatum L.). Sci. Hort. 120: 500-503.

Morgante M and Vogel J (1994). Compound Microsatellite Primers for the Detection of Genetic Polymorphisms. US Patent Application No. 08/326456.

Murray MG and Thompson WF (1980). Rapid isolation of high molecular weight plant DNA. Nucleic Acids Res. 8: 4321-4325.

Papp N, Szilvassy B, Abranko L, Szabo T, et al. (2010). Main quality attributes and antioxidants in Hungarian sour cherries: identification of genotypes with enhanced functional properties. Int. J. Food Sci. Technol. 45: 395-402.

Patil BR, Archak S, Gautam D and Salimath PM (2010). Narrow genetic base of private sector tomato varieties revealed by RAPD profiles. Electron. J. Plant Breed. 1: 1153-1158.

Penner GA, Bush A, Wise R, Kim W, et al. (1993). Reproducibility of random amplified polymorphic DNA (RAPD) analysis among laboratories. PCR Methods Appl. 2: 341-345.

Powell W, Morgante M, Andre C, Hanafey M, et al. (1996). The comparison of RFLP, RAPD, AFLP and SSR (microsatellite) markers for germplasm analysis. Mol. Breed. 2: 225-238.

Rajput SG, Wable KJ, Sharma KM, Kubde PD, et al. (2005). Reproducibility testing of RAPD and SSR markers in Tomato. Afr. J. Biotechnol. 5: 108-112.

Reddy PM, Saral N and Siddiq EA (2002). Inter simple sequence repeat (ISSR) polymorphism and its applications in plant breeding. Euphytica 128: 9-17.

Sonah H, Desmukh RK, Singh VP, Gupta DK, et al. (2011). Genomic resources in horticultural crops: status, utility and challenges. Biotechnol. Adv. 29: 199-209.

Sureja AK, Sirohi PS, Behera TK and Mohapatra T (2006). Molecular diversity and its relationship with hybrid performance and heterosis in ash gourd [Benincasa hispida (Thunb) Cogn.]. J. Hortic. Sci. Biotechnol. 81: 33-38.

Verma KVVK, Behera TK, Munshi AD, Parida SK, et al. (2007). Genetic diversity of ash gourd [Benincasa hispida (Thunb.) Cogn.] inbred lines based on RAPD and ISSR markers and their hybrid performance. Sci. Hort. 113: 231-237. 
Vishwanath K, Ananthararayanan TV, Pallavi HM, Ramegowda, et al. (2010). Varietal characterization of tomato cultivars based on RAPD markers. Res. J. Agric. Biol. Sci. 6: 713-715.

Vos P, Hogers R, Bleeker M, Reijans M, et al. (1995). AFLP: a new technique for DNA fingerprinting. Nucleic Acids Res. 23: 4407-4414.

Wang Y, Li X, Fang W, Wang C, et al. (2011). Genetic relationship analysis of flowering-mei using EST-SSR markers developed from apricot and fruiting-mei. Sci. Hort. 132: 12-17.

Welsh J and McClelland M (1990). Fingerprinting genomes using PCR with arbitrary primers. Nucleic Acids Res. 18: 7213-7218.

Williams JG, Kubelik AR, Livak KJ, Rafalski JA, et al. (1990). DNA polymorphisms amplified by arbitrary primers are useful as genetic markers. Nucleic Acids Res. 18: 6531-6535.

Williams MNV, Pande N, Nair M, Mohan M, et al. (1991). Restriction fragment length polymorphism analysis of polymerase chain reaction products amplified from mapped loci of rice (Oryza sativa L.) genomic DNA. Theor. Appl. Genet. 82: 489-498.

Zhang YX, Sun J, Zhang XR, Wang LH, et al. (2011). Analysis on genetic diversity and genetic basis of the main sesame cultivars released in China. Agric. Sci. China 10: 509-518.

Zhao MZ, Zhang YP, Wu WM, Wang C, et al. (2011). A new strategy for complete identification of 69 grapevine cultivars using random amplified polymorphic DNA (RAPD) markers. Afr. J. Plant Sci. 5: 273-280.

Zietkiewicz E, Rafalski A and Labuda D (1994). Genome fingerprinting by simple sequence repeat (SSR)-anchored polymerase chain reaction amplification. Genomics 20: 176-183. 